\title{
Characterizing Spot Price Dynamics in Public Cloud Environments
}

\author{
Bahman Javadi ${ }^{\mathrm{a}, *}$, Ruppa K. Thulasiram ${ }^{\mathrm{b}}$, Rajkumar Buyya ${ }^{\mathrm{c}}$ \\ ${ }^{a}$ School of Computing, Engineering and Mathematics \\ University of Western Sydney, Australia \\ ${ }^{b}$ Computational Financial Derivatives (CFD) Laboratory \\ Department of Computer Science \\ University of Manitoba, Winnipeg, Canada \\ ${ }^{c}$ Cloud Computing and Distributed Systems (CLOUDS) Laboratory \\ Department of Computing and Information Systems \\ The University of Melbourne, Australia
}

\begin{abstract}
The surge in demand for utilizing public Cloud resources has introduced many trade-offs between price, performance and recently reliability. Amazon's Spot Instances (SIs) create a competitive bidding option for the public Cloud users at lower prices without providing reliability on services. It is generally believed that SIs reduce monetary cost to the Cloud users, however it appears from the literature that their characteristics have not been explored and reported. We believe that characterization of SIs is fundamental in the design of stochastic scheduling algorithms and fault tolerant mechanisms in public Cloud environments for spot market. In this paper, we have done a comprehensive analysis of SIs based on one year price history in four data centers of Amazon's EC2. For this purpose, we have analyzed all different types of SIs in terms of spot price and the inter-price time (time between price changes) and determined the time dynamics for spot price in hour-in-day and day-of-week. Moreover, we have proposed a statistical model that fits well these two data series. The results reveal that we are able to model spot price dynamics as well as the inter-price time of each SI by the mixture of Gaussians distribution with three or four components. The proposed model is validated through extensive simulations, which demonstrate that our model
\end{abstract}

\footnotetext{
*Corresponding author. Telephone: +61-2-9685 9181; Fax: +61-2-9685 9245

Email address: b.javadi@uws.edu.au (Bahman Javadi)
} 
exhibits a good degree of accuracy under realistic working conditions.

Keywords: Cloud Computing; Spot Instances; Spot Price; Statistical Model;

\section{Introduction}

Due to the surge in demand for using utility computing systems like public Cloud resources, many trade-offs between price and performance have emerged. One particular type of Cloud service, which is known as Infrastructureas-as-Service (IaaS) provides raw computing with different capacity and storage in the form of Virtual Machines (VMs) with various prices on a pay-asyou-go basis. For instance, Amazon provides on-demand and reserved VM instances, which are associated with a fixed set price [1]. However, Amazon can increase or decrease these prices based on their own local policy. There are 64 different types of instances with various capacities and prices under two operating systems (i.e. 32 for Linux and 32 for Windows) which are made available by Amazon in four data centers as illustrated in Table 1 (sorted by their prices) ${ }^{1}$. In this Table, the prices are given for Linux operating system and the instances labeled with 'm1', 'm2', and 'c1' are standard, high-memory, and high-CPU instances, respectively.

In December 2009, Amazon released a new type of instances called Spot Instance (SI) to sell the idle time of Amazon's EC2 data centers [2]. The price of an SI, spot price, depends on the type of instance as well as VM demand within each data center. In fact, spot instances are an alternative to other two classes of instances which offer a low price but less reliable and competitive bidding option for the public Cloud users. Therefore, another aspect, reliability, has been added to the existing trade-offs to make utility computing systems more challenging than ever.

In order to utilize SIs, the Cloud users provide a bid which is the maximum price to be paid for an hour of usage. Whenever the current price of an SI is equal or less than the user bid, the instance is made available to the user. If the price of an SI becomes higher than the user's bid, out-of-bid event (failure), the $\operatorname{VM}(\mathrm{s})$ will be terminated by Amazon automatically and user does not pay for any partial hour. However, if the user terminates the running

\footnotetext{
${ }^{1}$ Amazon now has 7 data centers around the world, but the four major data centers are considered in this research.
} 
Table 1: Prices of on-demand instances in different data centers of Amazon (prices given in cents).

\begin{tabular}{|l||c|c|c|c|c|c|c|}
\hline Instances & us-west & us-east & eu-west & ap-southeast & EC2 Compute Unit & Memory (GB) & Storage (GB) \\
\hline m1.small & 9.5 & 8.5 & 9.5 & 9.5 & 1 & 1.7 & 160 \\
\hline c1.medium & 19 & 17 & 19 & 19 & 5 & 4 & 350 \\
\hline m1.large & 38 & 34 & 38 & 38 & 6.5 & 1.7 & 850 \\
\hline m2.xlarge & 57 & 50 & 57 & 57 & 8 & 15 & 420 \\
\hline m1.xlarge & 76 & 68 & 76 & 76 & 20 & 7 & 1690 \\
\hline c1.xlarge & 76 & 68 & 76 & 76 & 13 & 34.2 & 1690 \\
\hline m2.2xlarge & 114 & 100 & 14 & 114 & 26 & 68.4 & 1690 \\
\hline m2.4xlarge & 228 & 200 & 228 & 228 & & & \\
\hline
\end{tabular}

$\operatorname{VM}(\mathrm{s})$, she has to pay for the full hour. Amazon charges users per hour by the market price of the SI at the time of VM creation.

There are a number of works on how to utilize SIs to decrease the monetary cost of utility computing for Cloud users [3, 4, 5]. However, a thorough statistical analysis and modeling of SIs have not been appeared in the literature, the focus of our research in this study. In this paper, we provide a comprehensive analysis of all SIs in terms of spot price and the inter-price time (time between price changes) in four Amazon's data centers (i.e. uswest, us-east, eu-west, and ap-southeast). Moreover, we propose a statistical model to capture the volatile spot prices in Amazon's data centers. The main contributions of this paper are as follows:

- We provide statistical analysis for all SIs in Amazon's EC2 data centers. We also determine the time correlation in spot price in terms of hourin-day and day-of-week.

- We model spot price and the inter-price time of each SI with the mixture of Gaussians distribution. A model calibration algorithm is also proposed to deal with an observed price trend in the real price history.

- We validate and verify the accuracy of our proposed model through simulation under realistic working conditions.

We believe that results of this research would significantly helpful in the design of stochastic scheduling algorithms and fault tolerant mechanisms (e.g. checkpointing and replication algorithms) for spot market in public Cloud environments. In addition, although Amazon is the only provider of SIs at the moment, some researches have been conducted to analyze the 
free computing resource markets $[6,7]$. So, this model can be used by other resource providers that look forward to offer such a service in the near future.

The paper is structured as follows. In Section 2, we describe the processes that we model in this paper. We discuss related work in Section 3. We examine the pattern of spot price in Section 4. In Section 5, we present the global statistics for all SIs. We then illustrate distribution fitting for spot price and the inter-price time in Section 6. In Section 7, we propose an algorithm for model calibration. We discuss the validation of the proposed models through simulation in Section 8. In Section 9, we summarize our contributions and describe future directions.

\section{Modeling Approach}

In this section, we describe two variables that we are going to analyze and model. In Amazon's data centers, SIs have two variables (i.e. spot price and inter-price time) specified by the Cloud provider and one variable (user's bid) determined by users. In this study, we focus on the analysis and modeling of spot price and the inter-price time as two highly volatile system variables. These variables are illustrated in Figure 1 where $P_{i}$ is the price of an SI at time $t_{i}$. So, the inter-price time is defined as $T_{i}=t_{i+1}-t_{i}$. Therefore, the time series of spot price $\left(P_{i}\right)$ and the inter-price time $\left(T_{i}\right)$ are analyzed and modeled in the following sections.

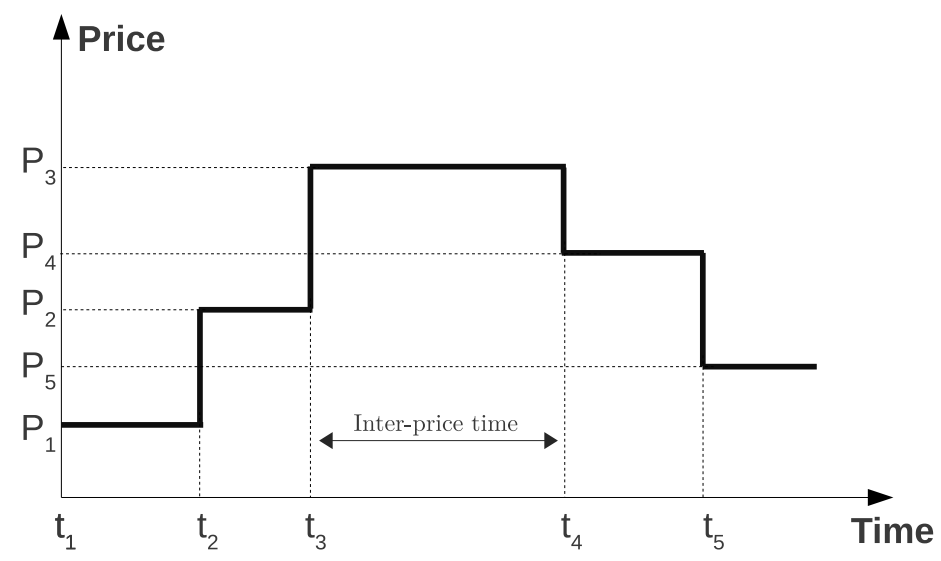

Figure 1: Spot price and the inter-price time of Spot instances.

The traces that we use in this study are one year price history of all Amazon SIs from the first of February 2010 to mid-February 2011. We use 
the first 10-month (Feb-2010 to Nov-2010) in the modeling process. These 10-month traces along with the last 2-month are used for the model validation purpose. The spot price history is freely provided by Amazon per SI for each data center and also available through other third-parties such as [8]. We do not use data prior to February 2010 due to an algorithm issue reported in [9] for prices. Moreover, we only use the SIs with Linux operating systems from all data centers. Due to similarity of the results, we present our findings for only two data centers (i.e. eu-west and us-east). Interested readers can refer to the extended version of this paper [10] for more discussions about other data centers.

\section{Related Work}

Although the current literature shows that SIs are good alternative for on-demand or reserve instances in terms of monetary cost, the characteristics of SIs are still not clear to users and researchers in the community. Wee [11] considered SIs as computing resources with real-time pricing. Focusing on the real price history of SIs, this paper concluded that still users need more monetary incentive to shift their workload into SIs. Another work that investigated the behavior of Spot prices is presented in [12], where authors used reverse engineering to construct a price model based on Auto-Regressive (AR) for SIs.

Our work is different in several aspects. We provide statistical analysis of all SIs and study their behavior in terms of hour-in-day and day-of-week. Moreover, we proposed to devise a statistical model for spot price as well as inter-price time. In addition, the simulation results reveal that we are able to model behavior of SIs by the mixture of Gaussian with three or four components.

In the following, we briefly review the other related work mainly investigated the usage of SIs to decrease the monetary cost of utility computing. Yi et al. [3, 4] introduced some checkpointing and migration mechanisms for reducing cost of SIs. They used the real price history of EC2 spot instances and showed how the adaptive checkpointing and migration schemes could decrease the monetary cost and improve the job completion times. Chaisiri et al. [13] proposed two provisioning algorithms based on stochastic programming, robust optimization, and sample-average approximation to optimized the provisioning cost for long-term and short-term planning. 
Moreover, in [14], a resource allocation policy to run deadline constrained jobs on SIs in a cost-effective manner is proposed.

In [15], a decision model for the optimization of performance, cost and reliability under SLA constraints while using SIs is proposed. They used the real price history and workload models to demonstrate how their proposed model can be used to bid optimally on SIs to reach different objective with desired levels of confidences. Mazzucco and Dumas [16] considered a case where a web service is deployed on SIs and proposed a bidding schema and resource allocation policies to optimize the web service provider's revenues.

Chohan et al. [17] proposed a method to utilize the SIs to speed up the MapReduce tasks. They provided a Markov chain to predict the expected lifetime of an SI. They concluded that having a fault tolerant mechanism is essential to run MapReduce jobs on SIs. Also, in [5], authors proposed a hybrid Cloud architecture to lease the SIs to manage peak loads of a local cluster. They proposed some provisioning policies and investigated the utilization of SIs compared to on-demand instances in terms of monetary cost saving and number of deadline violations.

Zhang et al. $[18,19]$ investigated dynamic market control problem in a single cloud provider motivated by the SIs offered by Amazon's EC2. They used static and dynamic optimizations for resource allocation to maximized the provider's revenue as well as user satisfactions. Rahman et al. [20] proposed resource allocation for Cloud users based on financial option theory to reduce the risk of dynamic price in spot markets. They showed that fluctuation in Amazon's SIs are much lower than expected values in a free market. This possibly is because of less users for SIs in compare to other other types of reliable resources such as on-demand instances.

Statistical modeling has been widely used in the characterization of computer systems' workloads and failures [21, 22, 23]. Although we apply the same techniques, the characteristics of SIs are far from the behavior of the workloads and failures, so require a comprehensive analysis.

\section{Patterns of Spot Price}

In this section, we examine hour-in-day and day-of-week time dynamics for the price of different SIs in eu-west and us-east data centers. We use the same approach as [24] to show how the price of one SI changes each hour in the day or each day of the week. As we have the price history in GMT time 
zone, we adjusted the local time for the time zone. This adjustment could reveal the dependency of spot price on the local time of a data center.

In Figure 2(a) and Figure 3(a), we create eight 3-hour time slots per day, and determine the average price of each SI in each time slot over all days. Then, we normalized this average by the maximum average price over all days. Note that the frequency of 3-hour sampling could be increased to 1hour sampling with 24 time slots in a day. However, it would only increase the sample size without shedding much light on the price dynamics, since spot price in Amazon's data centers are changing at the earliest every 2-3 hours (see Section 5). In these figures, we can observe that the y-axis is in the range of [0.98 1.0] where there is an increasing trend over the first-half of each day $\left(\left[\begin{array}{ll}0 & 12\end{array}\right]\right)$ and decreasing trend in spot price during the second-half of each day for all SIs in each data center.

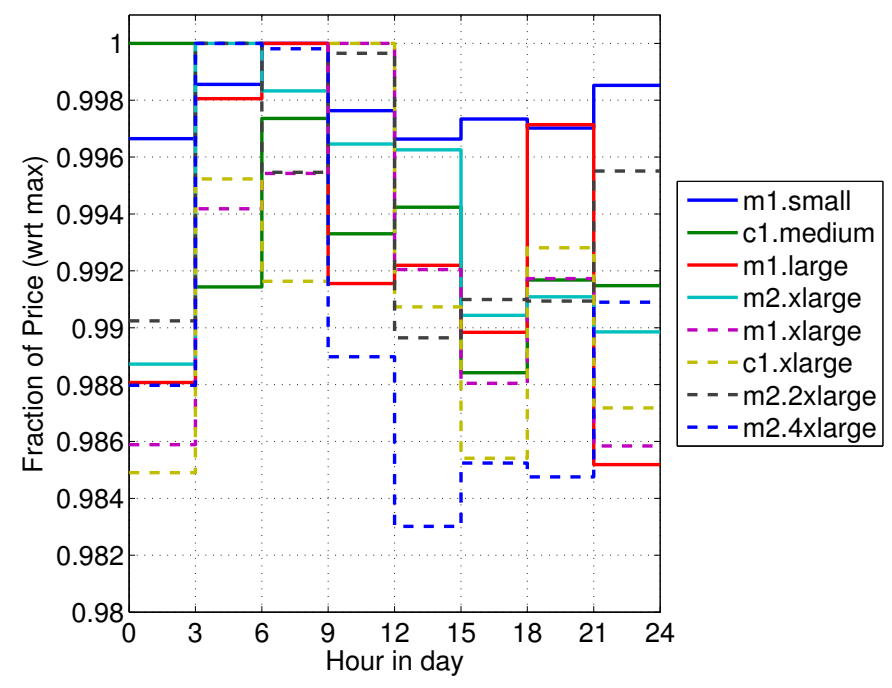

(a) Hour-in-day

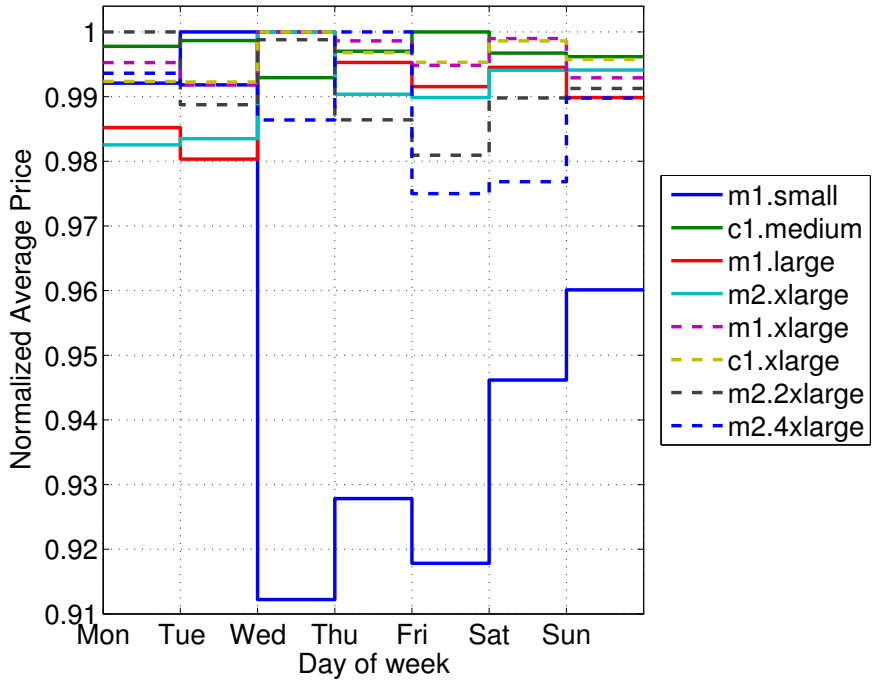

(b) Day-of-week

Figure 2: Patterns of spot price in eu-west data center.

In Figure 2(b) and Figure 3(b), we applied the same procedure to obtain the average price over seven 24-hours time slots within a week. The y-axis in these figures has wider range of $[0.911 .0]$ for eu-west and [0.95 1.0] for 


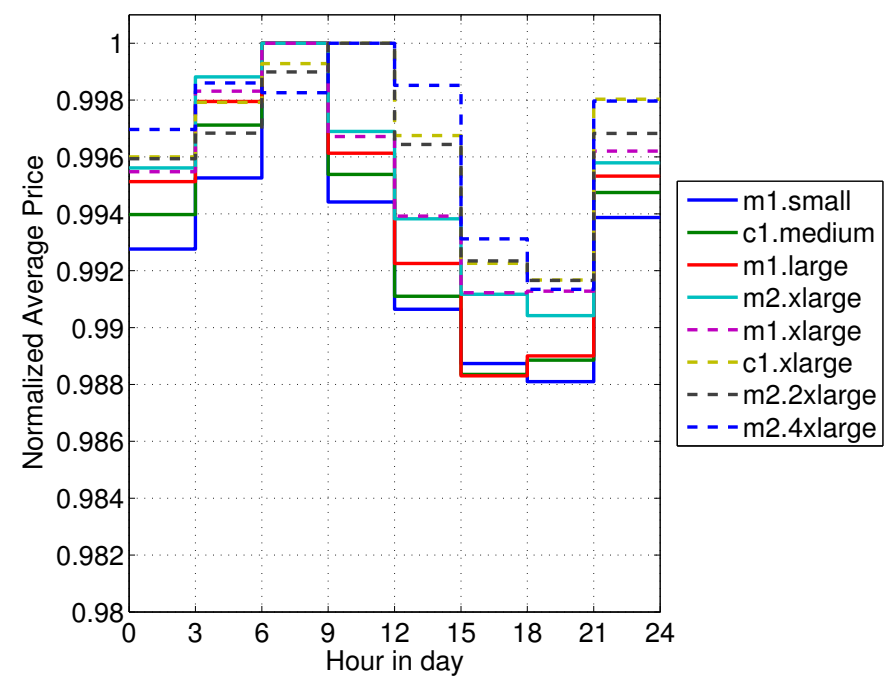

(a) Hour-in-day

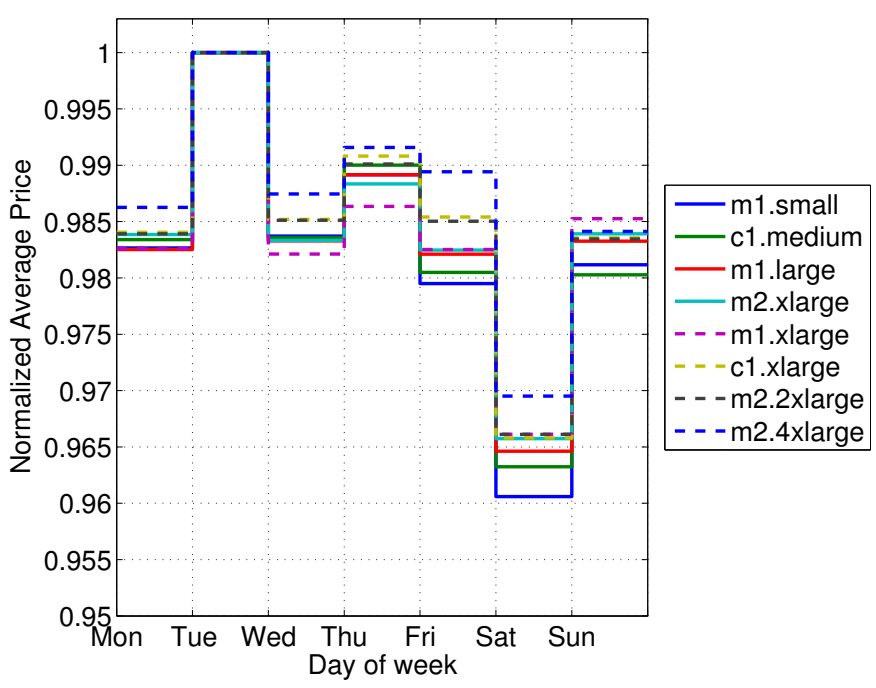

(b) Day-of-week

Figure 3: Patterns of spot price in us-east data center.

us-east data center ${ }^{2}$. As it is observable from this plot, we can not find any specific pattern for spot price in eu-west, except the decreasing in prices on weekends. However, for other Amazon's data centers such as us-east, we see more clear patterns in day of the week where on Tuesday we have the maximum price for almost all SIs in those data centers. Moreover, the lowest price are on Saturday, but on Sunday we again observe the increasing in price for all SIs.

\section{Global Statistics and Analysis}

In the following, we analyze the price history of different SIs in eu-west and us-east data centers. It has been shown that spot prices are tend to be random rather than market-driven [12]. So, analysis of global statistics can reveal some basic facts about $\mathrm{SIs}^{3}$.

\footnotetext{
${ }^{2}$ For other data centers, this range is ([0.95 1.0]).

${ }^{3}$ We conduct all of our statistical analysis using Matlab R2010b on a 32-bit Core2Duo $3.00 \mathrm{GHz}$ desktop with $3 \mathrm{~GB}$ of RAM. We use when possible standard tools provided by the
} 
Table 2: Statistics for spot price in eu-west data center (Values given in cents).

\begin{tabular}{|l||c|c|c|c|c|c|c|c|c|c|c|}
\hline Instances & Mean & TrMean & Median & Std & CV & IQR & Max & Min & Skewness & Kurtosis & No. \\
\hline m1.small & 4.00 & 4.00 & 4.00 & 0.19 & 0.05 & 0.20 & 9.50 & 3.80 & 9.44 & 242.97 & 3702 \\
\hline c1.medium & 8.00 & 8.00 & 8.00 & 0.27 & 0.03 & 0.40 & 10.10 & 7.60 & 0.28 & 3.91 & 3812 \\
\hline m1.large & 16.04 & 16.02 & 16.10 & 0.85 & 0.05 & 1.00 & 50.00 & 15.20 & 21.55 & 792.41 & 3875 \\
\hline m2.xlarge & 24.04 & 24.03 & 24.10 & 1.03 & 0.04 & 1.40 & 57.10 & 22.80 & 12.91 & 387.69 & 3763 \\
\hline m1.xlarge & 32.05 & 32.01 & 32.10 & 1.60 & 0.05 & 2.00 & 76.00 & 30.40 & 15.34 & 415.47 & 3917 \\
\hline c1.xlarge & 32.04 & 32.03 & 32.10 & 1.07 & 0.03 & 2.00 & 45.00 & 30.40 & 0.54 & 8.27 & 3658 \\
\hline m2.2xlarge & 56.04 & 56.04 & 56.20 & 1.83 & 0.03 & 3.42 & 76.00 & 53.20 & 0.25 & 4.99 & 4001 \\
\hline m2.4xlarge & 112.08 & 112.08 & 112.50 & 3.62 & 0.03 & 6.80 & 150.00 & 106.40 & 0.21 & 4.55 & 3912 \\
\hline
\end{tabular}

Table 3: Statistics for spot prices in us-east data center (Values given in cents).

\begin{tabular}{|l||c|c|c|c|c|c|c|c|c|c|c|}
\hline Instances & Mean & TrMean & Median & Std & CV & IQR & Max & Min & Skewness & Kurtosis & No. \\
\hline m1.small & 3.16 & 3.02 & 3.10 & 0.76 & 0.24 & 0.20 & 15.00 & 2.90 & 6.24 & 50.16 & 3279 \\
\hline c1.medium & 6.07 & 6.01 & 6.00 & 0.53 & 0.09 & 0.40 & 17.00 & 5.70 & 7.59 & 90.49 & 3643 \\
\hline m1.large & 12.98 & 12.15 & 12.10 & 4.47 & 0.34 & 0.70 & 68.00 & 11.40 & 6.62 & 60.29 & 2034 \\
\hline m2.xlarge & 17.78 & 17.05 & 17.10 & 4.87 & 0.27 & 1.10 & 80.00 & 16.20 & 7.09 & 57.62 & 3524 \\
\hline m1.xlarge & 24.18 & 24.05 & 24.10 & 2.56 & 0.11 & 1.50 & 100.00 & 22.80 & 22.03 & 599.91 & 3704 \\
\hline c1.xlarge & 26.01 & 24.26 & 24.20 & 8.68 & 0.33 & 1.60 & 128.00 & 22.80 & 4.85 & 27.78 & 3600 \\
\hline m2.2xlarge & 42.15 & 42.05 & 42.20 & 2.47 & 0.06 & 2.50 & 119.00 & 39.90 & 14.91 & 377.30 & 3790 \\
\hline m2.4xlarge & 84.58 & 84.04 & 84.20 & 8.46 & 0.10 & 5.00 & 240.00 & 79.80 & 13.54 & 218.92 & 3790 \\
\hline
\end{tabular}

We inspect the basic statistics of the traces in terms of spot price in Table 2 and Table 3 ; and in terms of the inter-price time in Table 4 and Table 5. The statistics in the tables are mean, trimmed mean (the mean value after discarding $10 \%$ of extreme values), median, standard deviation (Std), coefficient of variance (CV), interquartile range (IQR), maximum, minimum, skewness (the third moment), kurtosis (the forth moment) and number of samples.

These tables show three types of descriptive statistics. Statistics of the first type (mean, median, trimmed mean) reveal the central tendency of the distributions. The trimmed mean is a useful estimator of the central tendency as it is less sensitive to outliers. Statistics of the second type (CV, IQR, minimum, maximum) reflect the spread of the distributions. Statistics of the third type (kurtosis, skewness) represent the shape of the distributions.

First of all, we find that on average the price of SIs can be as low as

Statistical Toolbox. Otherwise, we implement or modify statistical functions ourselves. 
Table 4: Statistics for the inter-price time in eu-west data center (Values given in hours).

\begin{tabular}{|l||c|c|c|c|c|c|c|c|c|c|c|}
\hline Instances & Mean & TrMean & Median & Std & CV & IQR & Max & Min & Skewness & Kurtosis & No. \\
\hline m1.small & 1.96 & 1.61 & 1.35 & 2.66 & 1.35 & 0.30 & 109.08 & 0.02 & 19.94 & 727.54 & 3701 \\
\hline c1.medium & 1.91 & 1.59 & 1.34 & 1.86 & 0.97 & 0.32 & 22.81 & 0.02 & 4.53 & 30.63 & 3811 \\
\hline m1.large & 1.88 & 1.57 & 1.33 & 1.79 & 0.95 & 0.31 & 30.94 & 0.02 & 5.02 & 42.02 & 3874 \\
\hline m2.xlarge & 1.79 & 1.53 & 1.34 & 1.56 & 0.87 & 0.30 & 22.83 & 0.02 & 4.93 & 38.54 & 3762 \\
\hline m1.xlarge & 1.86 & 1.58 & 1.34 & 1.78 & 0.96 & 0.31 & 38.20 & 0.02 & 7.34 & 101.43 & 3916 \\
\hline c1.xlarge & 1.99 & 1.56 & 1.34 & 7.22 & 3.63 & 0.30 & 378.19 & 0.02 & 44.38 & 2169.40 & 3657 \\
\hline m2.2xlarge & 1.82 & 1.55 & 1.33 & 1.60 & 0.88 & 0.31 & 29.02 & 0.02 & 5.11 & 45.75 & 4000 \\
\hline m2.4xlarge & 1.86 & 1.58 & 1.34 & 1.71 & 0.92 & 0.31 & 26.51 & 0.02 & 5.20 & 44.28 & 3911 \\
\hline
\end{tabular}

$44 \%$ and $38 \%$ of on-demand instances for eu-west and us-east data centers, respectively. This expresses that there are some opportunities in reducing monetary cost of utility computing at the cost of unreliability. Moreover, the maximum price of some SIs (like m1.large) is bigger than the price of corresponding on-demand instance (specially in us-east data center). Since us-east is the cheapest data center, more users demand increases the fluctuation in spot prices (The higher value of CV in spot prices in us-east confirms this variability). Thus, even if the users' bid is as high as the on-demand prices, we may still have a probability of out-of-bid events.

The results in these tables reveal that the ratios between the mean and the median for spot price and the inter-price time of SIs are close to 1 for each trace. This indicates that Gaussian distribution might be a good option for the model. However, the skewness and kurtosis values show that the underlying distributions are right-skewed and short-tailed. Therefore, Gaussian distribution may not be a representative model to use and a better distribution is in order.

Additionally, we can observe that the inter-price time is more variable than spot price due to higher values of coefficient of variance. Also, analysis of the trimmed mean confirmed that inter-price time has greater variability. Therefore, we may need distributions with higher degrees of freedom, to model the inter-price time for these traces. It is worth noting that the minimum inter-price time is almost one hour in all data centers except euwest which is about a few minutes and can be seen in Table 4. Moreover, in eu-west and us-east data centers, the set price of SIs are stable on average for 2-3 hours. This observation is valid for other data centers as well [10]. This is the justification of 3-hour time slots to examine patterns of spot price in Figure 2(a) and Figure 3(a). 
Table 5: Statistics for the inter-price time in us-east data center (Values given in hours).

\begin{tabular}{|l||c|c|c|c|c|c|c|c|c|c|c|}
\hline Instances & Mean & TrMean & Median & Std & CV & IQR & Max & Min & Skewness & Kurtosis & No. \\
\hline m1.small & 2.22 & 1.66 & 1.36 & 3.53 & 1.59 & 0.32 & 76.59 & 0.78 & 9.21 & 130.29 & 3278 \\
\hline c1.medium & 2.00 & 1.65 & 1.37 & 2.09 & 1.05 & 0.31 & 49.91 & 1.00 & 6.91 & 98.48 & 3642 \\
\hline m1.large & 3.58 & 2.20 & 1.44 & 18.60 & 5.20 & 1.54 & 657.29 & 1.00 & 26.29 & 824.35 & 2033 \\
\hline m2.xlarge & 1.91 & 1.58 & 1.34 & 2.02 & 1.06 & 0.31 & 36.26 & 1.00 & 6.11 & 61.19 & 3523 \\
\hline m1.xlarge & 1.96 & 1.62 & 1.34 & 3.05 & 1.55 & 0.32 & 145.98 & 0.58 & 30.51 & 1370.41 & 3703 \\
\hline c1.xlarge & 2.02 & 1.66 & 1.35 & 3.38 & 1.67 & 0.33 & 171.62 & 1.00 & 35.74 & 1758.12 & 3599 \\
\hline m2.2xlarge & 1.92 & 1.62 & 1.34 & 1.94 & 1.01 & 0.31 & 50.40 & 1.01 & 8.42 & 143.99 & 3789 \\
\hline m2.4xlarge & 1.92 & 1.62 & 1.35 & 1.76 & 0.92 & 0.32 & 23.02 & 1.00 & 4.50 & 30.98 & 3789 \\
\hline
\end{tabular}

\section{Distribution Fitting}

After global statistical analysis, we first inspect the Probability Density Function (PDF) of spot price and the inter-price time. Then, we conduct parameter fitting for the Mixture of Gaussians (MoG) distribution by the expectation maximization (EM) algorithm to model both time series. We considered other distributions, such as Weibull, Normal, Log-normal and Gamma distributions as well. However, the mixture of Gaussians distribution shows the better fit with respect to others [10]. In this section, we show the process of fitting for eu-west data center to avoid presenting similar figures and plots. We present the final results for both selected data centers.

\subsection{Probability Densities}

The PDFs of spot price of each SI in eu-west data center are depicted in Figure 4. We can easily observe bi-modality in the probability density functions. Moreover, the price distribution of all SIs, except m1.small, are almost symmetric. The exception for m1.small is possibly because of diverse usage patterns of this instance as the cheapest resource in each data center.

The PDFs of the inter-price time for each SI in eu-west are represented in Figure 5. Obviously, there is a single dominant mode (peak) in the density functions when compared to (nearly) equal peaks in the PDFs of spot price. Most of SIs have the peak around two hours, which confirm the results of the previous section (see Mean column in Table 4). The reason for the very sharp peak in these density functions is investigated in Section 7. Observation from the plotted density functions of both time series, our decision to propose a mixture of Gaussians distribution as a good candidate for approximating such density shapes is further strengthened. This is also confirmed by [26] 


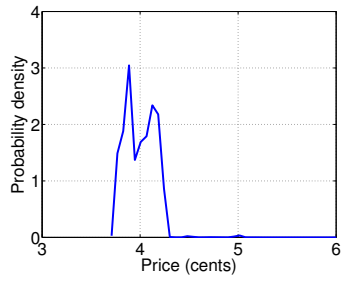

(a) m1.small

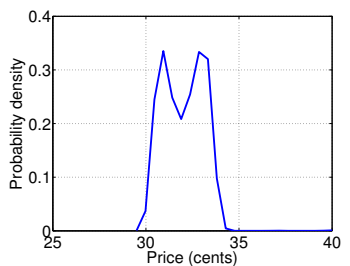

(e) m1.xlarge

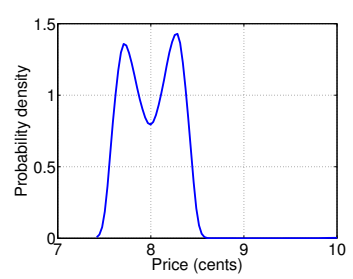

(b) c1.medium

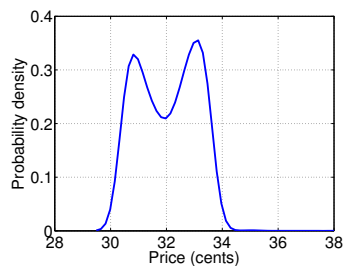

(f) c1.xlarge

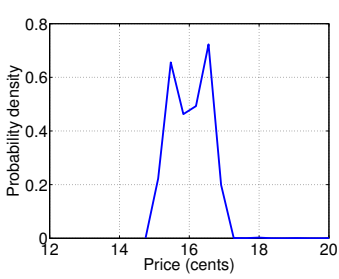

(c) m1.large

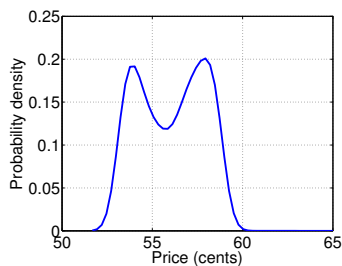

(g) m2.2xlarge

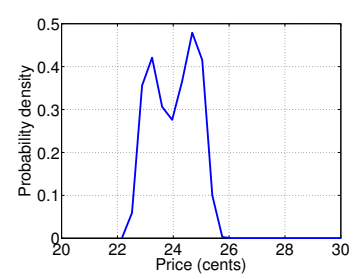

(d) m2.xlarge

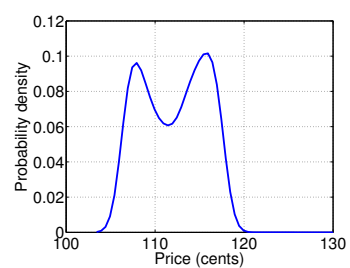

(h) m2.4xlarge

Figure 4: Probability density functions of spot price for all SIs in eu-west data center.

where they used a mixture of Gaussians distribution to model a multi-modal density function.

\subsection{Parameter Estimation and Goodness of Fit Tests}

In this section, we conduct parameter fitting for the mixture of Gaussians distribution with $k$ components, which is defined as follows:

$$
c d f\left(x ; k, \vec{p}, \vec{\mu}, \overrightarrow{\sigma^{2}}\right)=\sum_{i=1}^{k} \frac{p_{i}}{2}\left(1+\operatorname{erf}\left(\frac{x-\mu_{i}}{\sigma_{i} \sqrt{2}}\right)\right)
$$

where $\vec{\mu}, \overrightarrow{\sigma^{2}}$, and $\vec{p}$ are the vector of mean, variance and probability of components with $k$ items. Also, erf () is the error function, which is defined as follows:

$$
\operatorname{erf}(x)=\frac{2}{\sqrt{\pi}} \int_{0}^{x} e^{-t^{2}} d t
$$

To maximize the data likelihood in terms of parameters $\vec{\mu}$ and $\overrightarrow{\sigma^{2}}$ where $k$ is given a priori, we adopt the expectation maximization (EM) algorithm, 


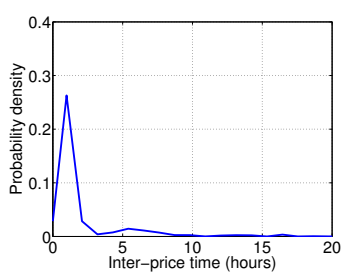

(a) m1.small

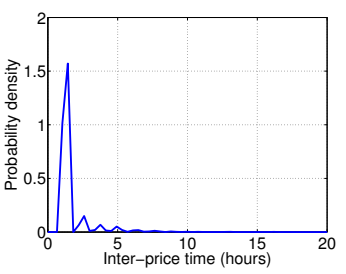

(e) m1.xlarge

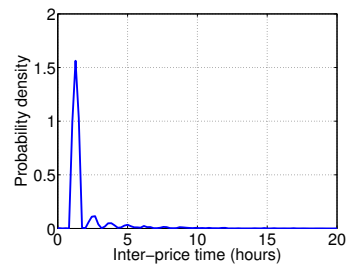

(b) c1.medium

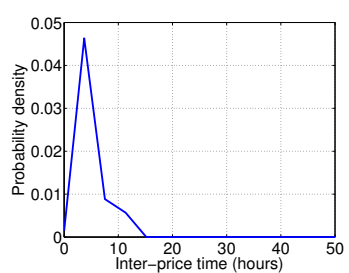

(f) c1.xlarge

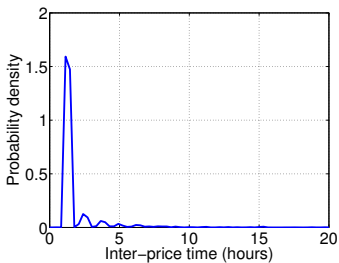

(c) m1.large

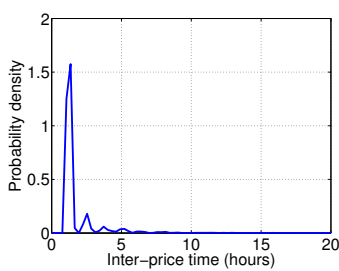

(g) m2.2xlarge

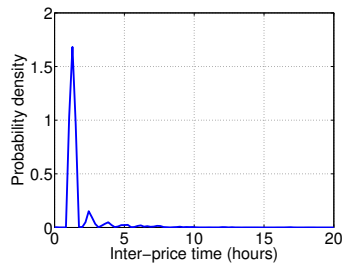

(d) m2.xlarge

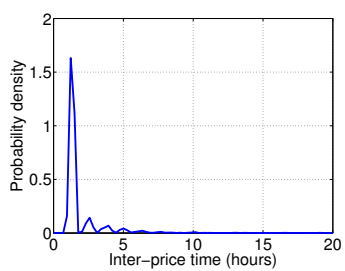

(h) m2.4xlarge

Figure 5: Probability density functions of the inter-price time for all SIs in eu-west data center.

which is a general maximum likelihood estimation [21]. Parameter fitting was done using Model Based Clustering (MBC), which is introduced by Fraley and Raftery [25]. MBC is a methodological framework that can be used for data clustering as well as (multi)variate density estimation. One assumption is that data has several components each of which is generated by a probability distribution. Model Based Clustering uses Bayesian model selection to choose the best model in terms of number of components [26]. In contrast, we use the goodness of fit (GOF) tests to determine the best model as we have an estimation for the number of components in the model. We choose the number of components between 2 and $4(2 \leq k \leq 4)$ based on the observation of the density functions. We measured the goodness of fit of the resulting models using a visual method (i.e. standard probability-probability (PP) plots) and Kolmogorov-Smirnov (KS) and Anderson-Darling (AD) tests [21] as quantitative metrics.

After parameter estimation, we must examine the quality of each fit through GOF tests. First of all, we present the graphical results of distribution fitting for spot price and the inter-price time of all SIs in Figure 6 

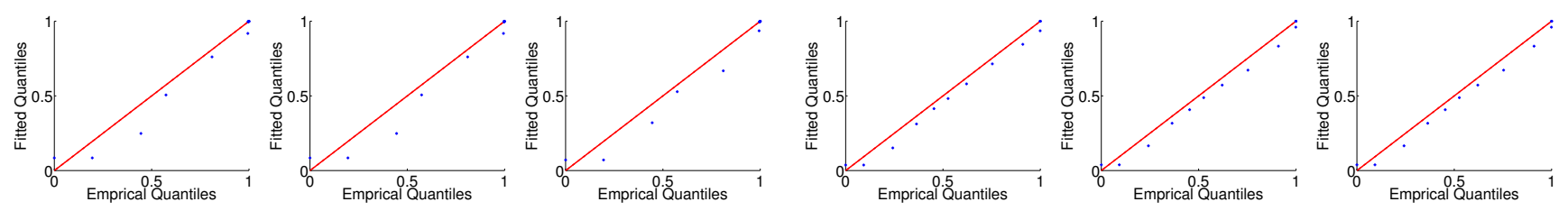

(a) m1.small

(b) c1.medium
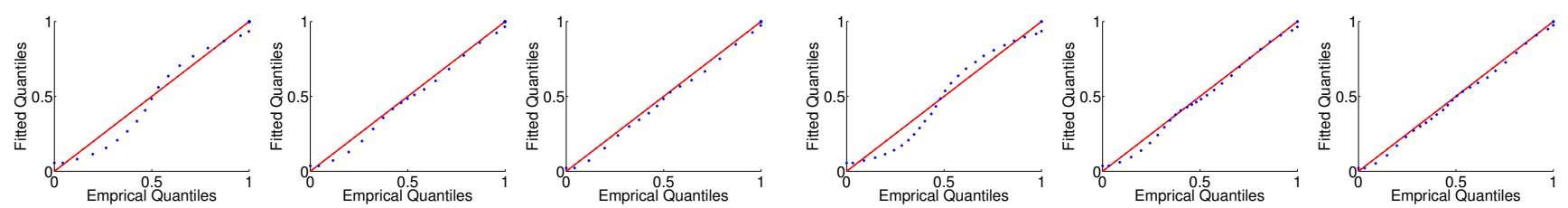

(c) m1.large

(d) m2.xlarge
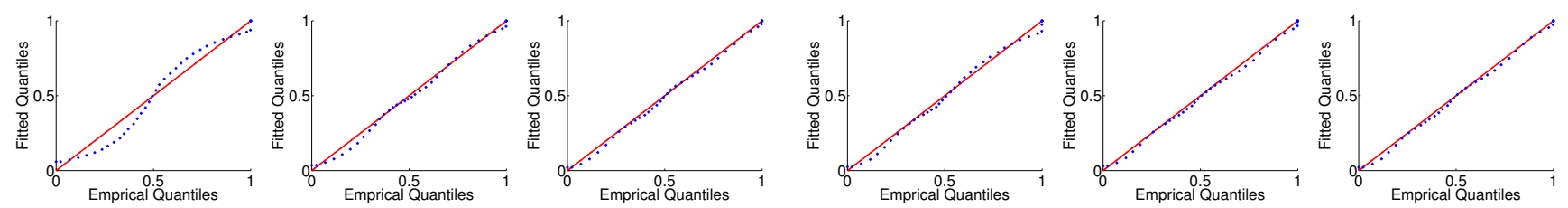

(e) m1.xlarge

(f) c1.xlarge
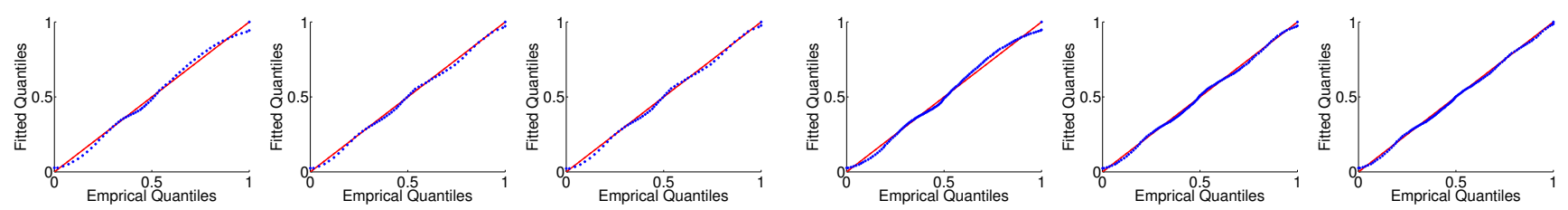

(g) m2.2xlarge

(h) m2.4xlarge

Figure 6: PP-plots of spot price in eu-west for mixture of Gaussians $(k=2, k=3, k=4)$. $\mathrm{X}$-axis: empirical quantiles, and Y-axis: fitted quantiles. 
and Figure 7 for eu-west data center, respectively. In these plots, the closer the plots are to the line $y=x$, the better the fit. In each plot $\mathrm{x}$-axis is the empirical quantiles while $\mathrm{y}$-axis is the fitted quantiles. Based on these figures, the mixture of Gaussians distribution with three or four components can fit spot price and the inter-price time of SIs in eu-west data center. The only instance which is hard to fit, specially in terms of spot price, is m1.small instance.

To be more quantitative, we also report the p-values of two GOF tests (i.e. KS and AD tests). We randomly select a subsample of 50 of each trace and compute the p-values iteratively for 1000 times and finally obtain the average p-value. This method is similar to the one used by the authors in [27]. Moreover, in all cases the coefficient of variance is less than one (i.e., $C V<1$ ), so the average value is a representative estimate.

The results of GOF tests are listed in Table 6 and Table 7 for spot price in eu-west and us-east data centers. For the inter-price time, the p-values are presented in Table 8 and Table 9 in eu-west and us-east, respectively. Moreover, in each row the best fits are highlighted. In some cases, we have two winners as there is one best fit per each GOF test. These quantitative results strongly confirm the graphical results of the PP-plots. The p-values in the first row of Table 6 and Table 7 express that spot price of m1.small instance is hard to fit, even with four components.

The set of parameters for MoG distributions are listed in Table 10 and Table 11 for spot price and the inter-price time for $k=3$ in eu-west and useast data center, respectively. It is worth noting that in the list of parameters, we just report two items of parameter $\vec{p}$, as the last item in this vector can be computed using others. (i.e. $p_{k}=1-\sum_{i=1}^{k-1} p_{i}$ ).

As the number of parameters in the MoG distribution is $3 k+1$ (see Equation 1), so we have a trade-off between accuracy and complexity of the model. With fewer components, the analysis becomes simpler that gives reasonably good fit to spot price and inter-price time with a compromise of accuracy to some extent. This would significanly help in understanding the data series on the first step. With this understanding a model to better fit the data series with many components can be designed. Hence, for the sake of simplicity and homogeneity, in the rest of this paper we choose the model with three components $(k=3)$ for both spot price and the inter-price time for further analysis. The set of parameters for MoG distributions for spot price and the inter-price time for $2 \leq k \leq 4$ in all data centers are reported in [10]. 

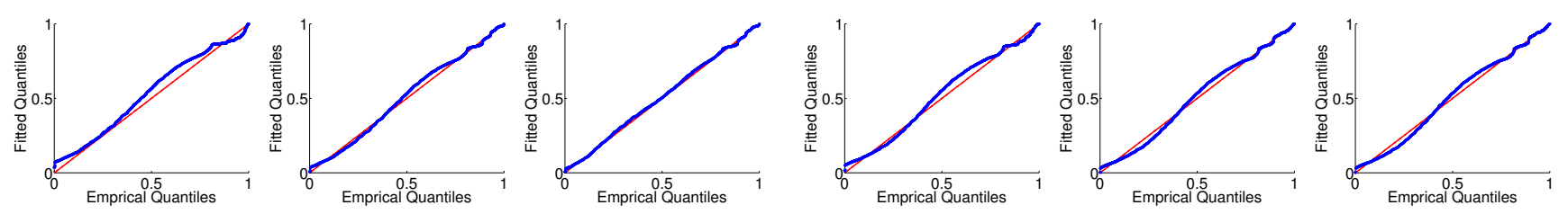

(a) m1.small

(b) c1.medium
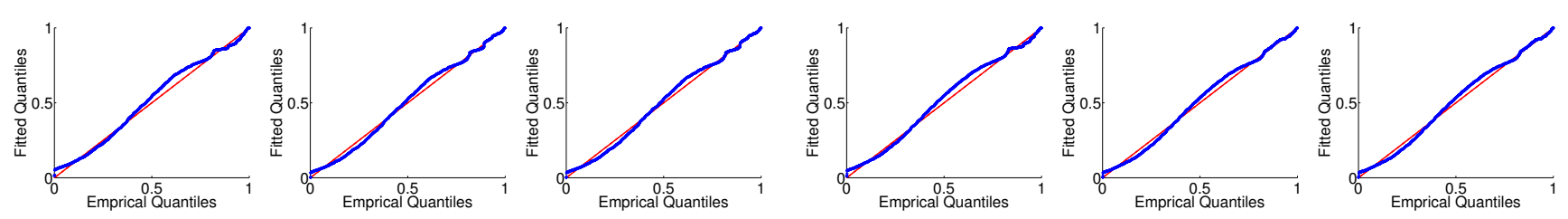

(c) m1.large

(d) m2.xlarge
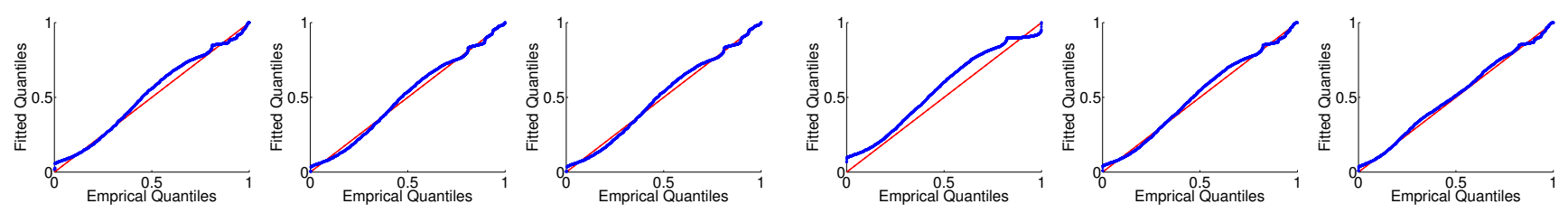

(e) m1.xlarge

(f) c1.xlarge
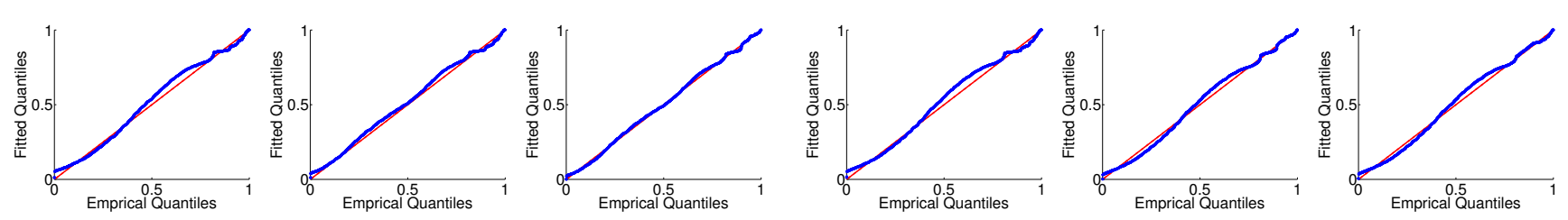

(g) m2.2xlarge

(h) m2.4xlarge

Figure 7: PP-plots of the inter-price time in eu-west for mixture of Gaussians $(k=2, k=3$, $k=4)$. X-axis: empirical quantiles, and Y-axis: fitted quantiles. 
Table 6: p-values resulting from KS and $\mathrm{AD}$ tests for spot price in eu-west.

\begin{tabular}{|l||c|c|c|}
\hline Instances & MoG $(k=2)$ & MoG $(k=3)$ & MoG $(k=4)$ \\
\hline m1.small & 0.0160 .791 & 0.0170 .789 & 0.0530 .803 \\
\hline c1.medium & 0.2110 .779 & 0.2170 .791 & 0.2240 .790 \\
\hline m1.large & 0.1130 .678 & 0.3190 .752 & 0.3540 .754 \\
\hline m2.xlarge & 0.1390 .616 & 0.3560 .721 & 0.4150 .734 \\
\hline m1.xlarge & 0.1340 .570 & 0.3690 .708 & 0.4310 .706 \\
\hline c1.xlarge & 0.3940 .681 & 0.4440 .705 & 0.4210 .707 \\
\hline m2.2xlarge & 0.4200 .648 & 0.4690 .682 & 0.4500 .672 \\
\hline m2.4xlarge & 0.4290 .617 & 0.4630 .637 & 0.4760 .653 \\
\hline
\end{tabular}

Table 7: p-values resulting from KS and AD tests for spot price in us-east.

\begin{tabular}{|c|c|c|c|}
\hline Instances & MoG $(k=2)$ & MoG $(k=3)$ & MoG $(k=4)$ \\
\hline m1.small & 0.0000 .732 & $0.000 \quad 0.736$ & $\begin{array}{lll}0.000 & 0.727\end{array}$ \\
\hline c1.medium & 0.0560 .774 & 0.1500 .796 & 0.1470 .797 \\
\hline m1.large & 0.1580 .726 & 0.1570 .723 & 0.3290 .763 \\
\hline m2.xlarge & 0.1320 .697 & $0.138 \quad 0.690$ & 0.1260 .693 \\
\hline m1.xlarge & $0.142 \quad 0.634$ & $\begin{array}{lll}0.138 & 0.633\end{array}$ & $\begin{array}{lll}0.142 & 0.627\end{array}$ \\
\hline c1.xlarge & 0.1800 .669 & 0.1870 .673 & 0.1870 .673 \\
\hline $\mathrm{m} 2.2 x$ large & 0.1690 .553 & 0.4330 .693 & 0.4530 .699 \\
\hline m2.4xlarge & 0.1690 .464 & 0.1810 .470 & 0.1810 .46 \\
\hline
\end{tabular}

Table 8: p-values resulting from KS and AD tests for the inter-price in eu-west.

\begin{tabular}{|l||c|c|c|}
\hline Instances & MoG $(k=2)$ & MoG $(k=3)$ & MoG $(k=4)$ \\
\hline m1.small & 0.3470 .476 & 0.4150 .592 & 0.4890 .627 \\
\hline c1.medium & 0.3820 .546 & 0.3900 .566 & 0.3800 .566 \\
\hline m1.large & 0.3900 .552 & 0.3870 .573 & 0.4000 .574 \\
\hline m2.xlarge & 0.3890 .556 & 0.3930 .566 & 0.4050 .585 \\
\hline m1.xlarge & 0.3690 .526 & 0.3910 .564 & 0.4060 .581 \\
\hline c1.xlarge & 0.2210 .319 & 0.3990 .561 & 0.4670 .602 \\
\hline m2.2xlarge & 0.3760 .532 & 0.4260 .570 & 0.4630 .610 \\
\hline m2.4xlarge & 0.3680 .529 & 0.3830 .569 & 0.3950 .573 \\
\hline
\end{tabular}


Table 9: p-values resulting from KS and AD tests for the inter-price in us-east.

\begin{tabular}{|l||c|c|c|}
\hline Instances & MoG $(k=2)$ & MoG $(k=3)$ & MoG $(k=4)$ \\
\hline m1.small & 0.3600 .467 & 0.4330 .592 & 0.4760 .623 \\
\hline c1.medium & 0.3810 .517 & 0.4410 .598 & 0.4890 .622 \\
\hline m1.large & 0.0040 .052 & 0.3290 .508 & 0.4110 .595 \\
\hline m2.xlarge & 0.3700 .528 & 0.3730 .563 & 0.4640 .617 \\
\hline m1.xlarge & 0.2720 .389 & 0.4010 .569 & 0.3910 .562 \\
\hline c1.xlarge & 0.2400 .341 & 0.3960 .570 & 0.4600 .597 \\
\hline m2.2xlarge & 0.3530 .498 & 0.4010 .579 & 0.4590 .605 \\
\hline m2.4xlarge & 0.3810 .537 & 0.4340 .569 & 0.4020 .578 \\
\hline
\end{tabular}

Table 10: Parameters of the mixture of Gaussians distributions for spot price and interprice time in eu-west.

\begin{tabular}{|c|c|c|c|c|c|c|}
\hline \multirow[t]{2}{*}{ Instances } & \multicolumn{3}{|c|}{ Price model $(k=3)$} & \multicolumn{3}{|c|}{ Inter-price model $(k=3)$} \\
\hline & $\vec{p}$ & $\vec{\mu}$ & $\overrightarrow{\sigma^{2}}$ & $\vec{p}$ & $\vec{\mu}$ & $\overrightarrow{\sigma^{2}}$ \\
\hline m1.small & 0.0030 .003 & $5.216 \quad 5.216 \quad 3.997$ & $1.6701 .670 \quad 0.020$ & 0.1780 .028 & $3.474 \quad 11.536 \quad 1.292$ & $2.308 \quad 120.051 \quad 0.022$ \\
\hline c1.medium & 0.4430 .276 & $8.018 \quad 8.292 \quad 7.703$ & $0.0450 .006 \quad 0.006$ & 0.8070 .090 & 1.2796 .4522 .876 & $0.022 \quad 12.435 \quad 0.528$ \\
\hline m1.large & 0.4920 .505 & $15.556 \quad 16.470 \quad 24.401$ & $\begin{array}{llll}0.059 & 0.048 & 114.879\end{array}$ & 0.0680 .126 & $6.793 \quad 3.040 \quad 1.276$ & $13.803 \quad 0.940 \quad 0.022$ \\
\hline m2.xlarge & 0.4450 .001 & 23.26453 .50024 .643 & $0.10912 .960 \quad 0.135$ & 0.8240 .066 & 1.2842 .5065 .166 & $0.022 \quad 0.035 \quad 8.192$ \\
\hline m1.xlarge & 0.4570 .002 & $31.01053 .803 \quad 32.848$ & $0.184326 .523 \quad 0.249$ & 0.7930 .177 & 1.2833 .3108 .356 & 0.0221 .86429 .730 \\
\hline c1.xlarge & $0.261 \quad 0.243$ & $\begin{array}{llll}33.188 & 30.756 & 32.057\end{array}$ & $\begin{array}{llll}0.072 & 0.058 & 0.722\end{array}$ & 0.8110 .187 & $1.2864 .048 \quad 84.430$ & $0.0225 .341 \quad 15636.817$ \\
\hline $\mathrm{m} 2.2 \mathrm{xlarge}$ & $0.492 \quad 0.252$ & 56.11953 .78458 .100 & $\begin{array}{lll}1.813 & 0.157 & 0.216\end{array}$ & $0.405 \quad 0.399$ & $1.155 \quad 1.398 \quad 4.044$ & 0.0070 .0086 .795 \\
\hline $\mathrm{m} 2.4 x$ large & $0.263 \quad 0.249$ & $116.126 \quad 107.609112 .183$ & $0.898 \quad 0.660 \quad 7.061$ & 0.0630 .137 & $6.7053 .001 \quad 1.279$ & $13.5240 .863 \quad 0.022$ \\
\hline
\end{tabular}

Table 11: Parameters of the mixture of Gaussians distributions for spot price and interprice time in us-east

\begin{tabular}{|c|c|c|c|c|c|c|}
\hline \multirow[t]{2}{*}{ Instances } & \multicolumn{3}{|c|}{ Price model $(k=3)$} & \multicolumn{3}{|c|}{ Inter-price model $(k=3)$} \\
\hline & $\vec{p}$ & $\vec{\mu}$ & $\overrightarrow{\sigma^{2}}$ & $\vec{p}$ & $\vec{\mu}$ & $\overrightarrow{\sigma^{2}}$ \\
\hline m1.small & 0.0240 .952 & 6.0093 .0126 .009 & 3.4020 .0093 .402 & 0.1640 .043 & $3.581 \quad 13.935 \quad 1.301$ & $2.638 \quad 120.2120 .025$ \\
\hline c1.medium & 0.4390 .537 & 5.8086 .1678 .726 & 0.0070 .0112 .935 & 0.7800 .145 & 1.3012 .9547 .379 & $0.023 \quad 0.81420 .600$ \\
\hline $\mathrm{m} 1$. large & 0.5960 .094 & $11.979 \quad 22.345 \quad 12.066$ & $0.147 \quad 114.857 \quad 0.148$ & 0.0230 .389 & $\begin{array}{llll}54.787 & 3.976 & 1.277\end{array}$ & $11982.593 \quad 4.795 \quad 0.022$ \\
\hline $\mathrm{m} 2 . x$ large & 0.4610 .504 & $17.020 \quad 17.02038 .737$ & $0.299 \quad 0.299209 .027$ & 0.1470 .814 & $3.508 \quad 1.2829 .249$ & $1.809 \quad 0.023 \quad 27.356$ \\
\hline m1.xlarge & 0.0080 .439 & 41.51124 .04524 .023 & $\begin{array}{llll}425.466 & 0.591 & 0.593\end{array}$ & $0.778 \quad 0.015$ & $1.278 \quad 13.908 \quad 3.655$ & $0.022353 .873 \quad 3.005$ \\
\hline c1.xlarge & 0.0710 .328 & 51.72224 .06424 .028 & 340.1200 .5940 .593 & 0.7590 .016 & $1.280 \quad 14.298 \quad 3.651$ & $0.021459 .583 \quad 3.196$ \\
\hline $\mathrm{m} 2.2 \mathrm{x}$ large & 0.4440 .549 & 40.71543 .10461 .120 & $0.308 \quad 0.462 \quad 334.756$ & 0.0410 .778 & $8.230 \quad 1.278 \quad 3.230$ & $29.753 \quad 0.021 \quad 1.354$ \\
\hline $\mathrm{m} 2.4 \mathrm{xlarge}$ & 0.5940 .007 & $83.823 \quad 166.323 \quad 84.275$ & 7.0632453 .5276 .942 & 0.2180 .398 & $4.1941 .167 \quad 1.407$ & $\begin{array}{llll}7.461 & 0.008 & 0.007\end{array}$ \\
\hline
\end{tabular}




\section{Model Calibration}

In this section, we look into the time evolution of spot price and the interprice time, which potentially can lead us to obtain a more accurate model. For this purpose, we examine the scatter plot of spot price and the inter-price time during February 2010 till November 2010. We just present the plots for $\mathrm{m} 2$.4xlarge instance, as the results are consistent for other instance types within the data centers.

Figure 8(a) depicts the scatter plot of spot price for m2.4xlarge in euwest data center for the duration of the price history. As it can be seen in this figure, there is no clear correlation in spot price where they are evenly distributed in a specific range (this range depends on the type of instances). However, congestion of spot price is increased after mid-July and this is the case for all SIs in eu-west data center. To confirm this observation, we examine the scatter plot of the inter-price time for this SI in Figure 8(b). We observe that inter-price time become suddenly shorter after mid-July. That means, the frequency of changing price is increased while spot price remains bounded within a small price range. The inspection of other SIs within the data center reveals the same result. This is also the reason of very sharp peak in density functions of the inter-price time in Figure 5.

This trend is possibly due to some fine tunings made by Amazon in their pricing algorithm. It is worth noting that the same issue has been observed in other Amazon's EC2 data centers in different dates. As illustrated in Figure 9, this phenomenon is observable in us-east at the end of July 2010 for m2.4xlarge instances. Also, for us-west and ap-southeast data centers this changes happened in January 2011 (Figures are plotted in [10]).

Focusing on the scatter plot of the inter-price time (MoG model for $k=3$ ) presented in Figure 8(b), we can see that after mid-July only one component (i.e. component 3) remains and other components collapsed to a small band. As this observation is consistent over all SIs, we propose a model calibration algorithm (Algorithm 1) to find the date of collapsing (which is called calibration date) as well as remaining component(s).

The algorithm needs the trace of the inter-price time of an SI $\left(\right.$ Trace $\left._{\text {inst }}\right)$ and the number of components $(k)$. The result of mixture of Gaussians model with $k$ components is $\overrightarrow{i n d e x}$. Also, $\overrightarrow{\text { date }}$ is a vector, each element of which correspond to each item of $\overrightarrow{i n d e x}$. At first, the algorithm computes the probability of each component in each month in the whole trace and after that finds a list $\left(\overrightarrow{Q_{m}}\right)$ where the probability of one or more components is 


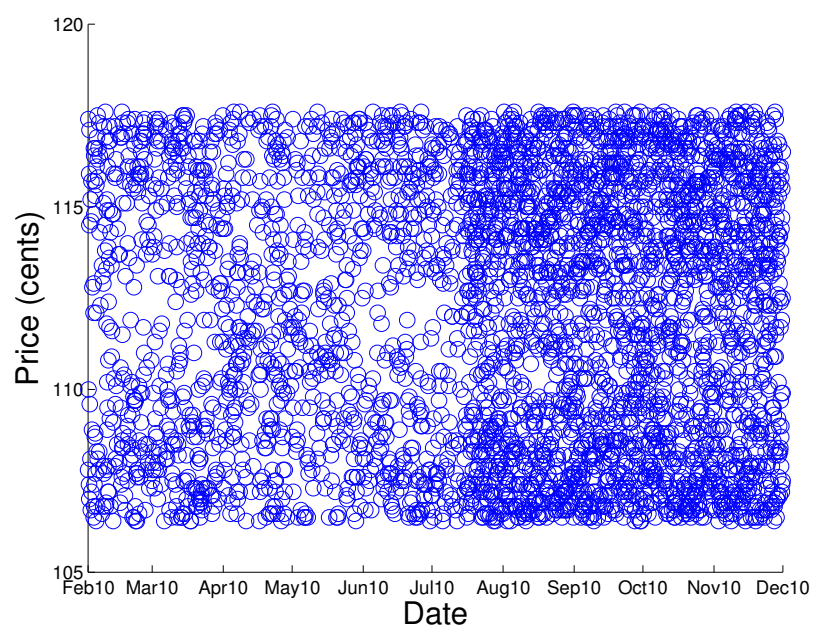

(a) Scatter plot of spot price for m2.4xlarge.

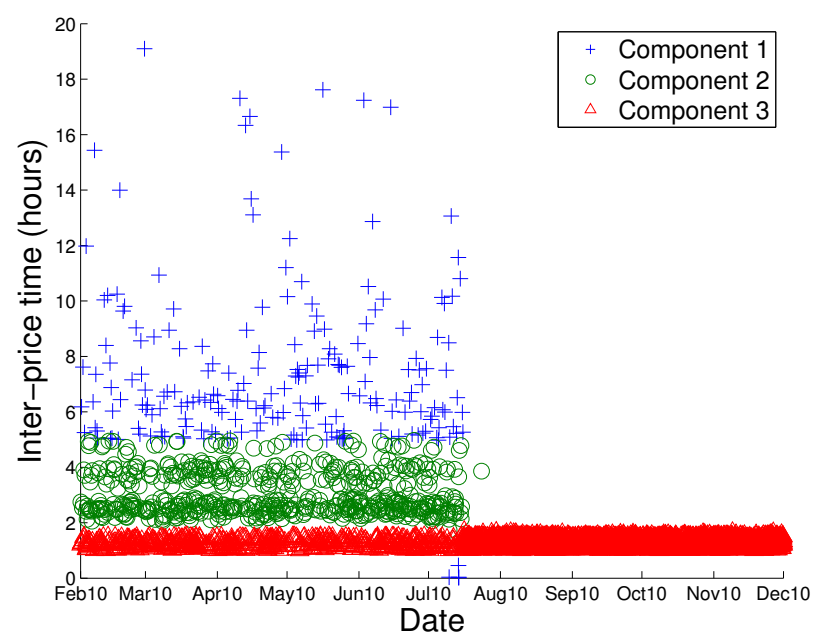

(b) Scatter plot along with the components' distribution of the inter-price time for m2.4xlarge.

Figure 8: Scatter plot of spot price and the inter-price time for m2.4xlarge in eu-west. 


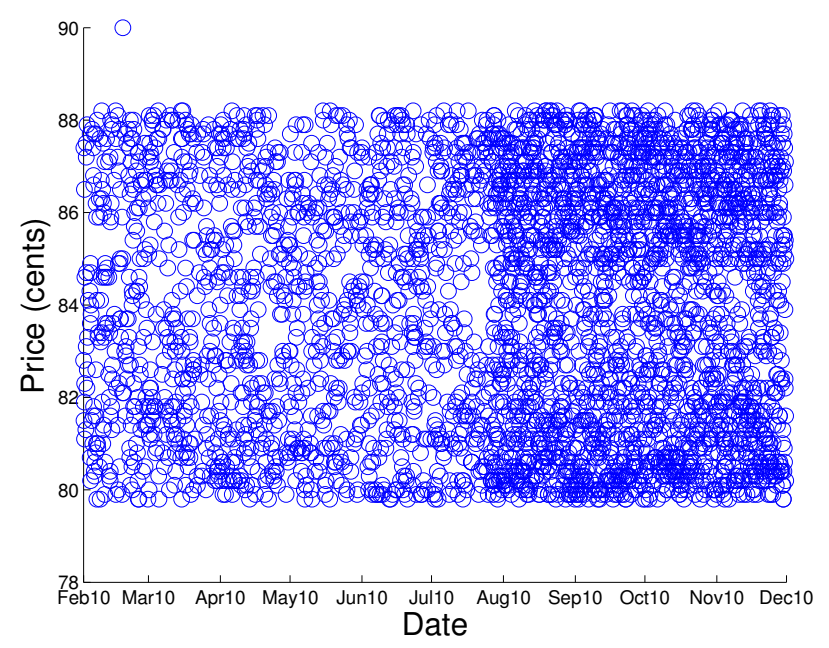

(a) Scatter plot of spot price for m2.4xlarge.

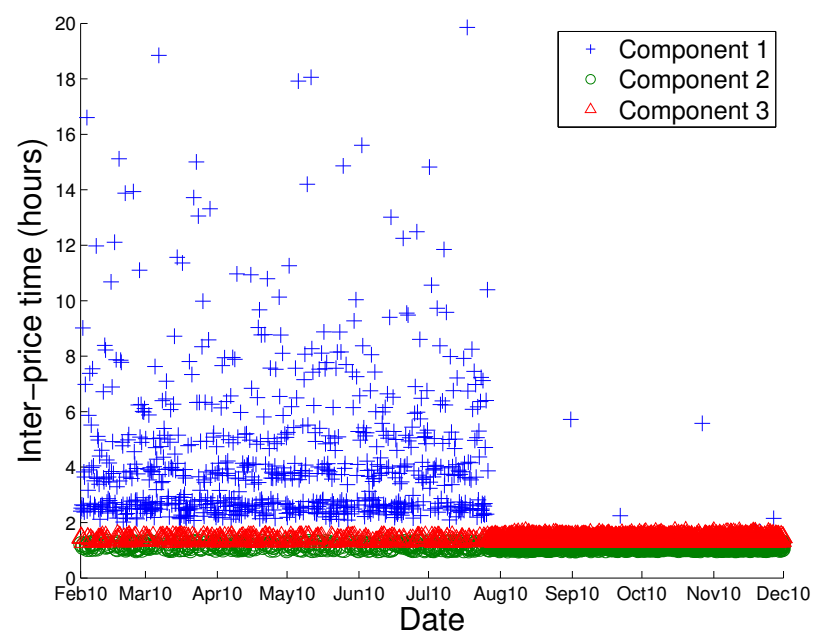

(b) Scatter plot along with the components' distribution of the inter-price time for m2.4xlarge.

Figure 9: Scatter plot of spot price and the inter-price time for m2.4xlarge in us-east. 


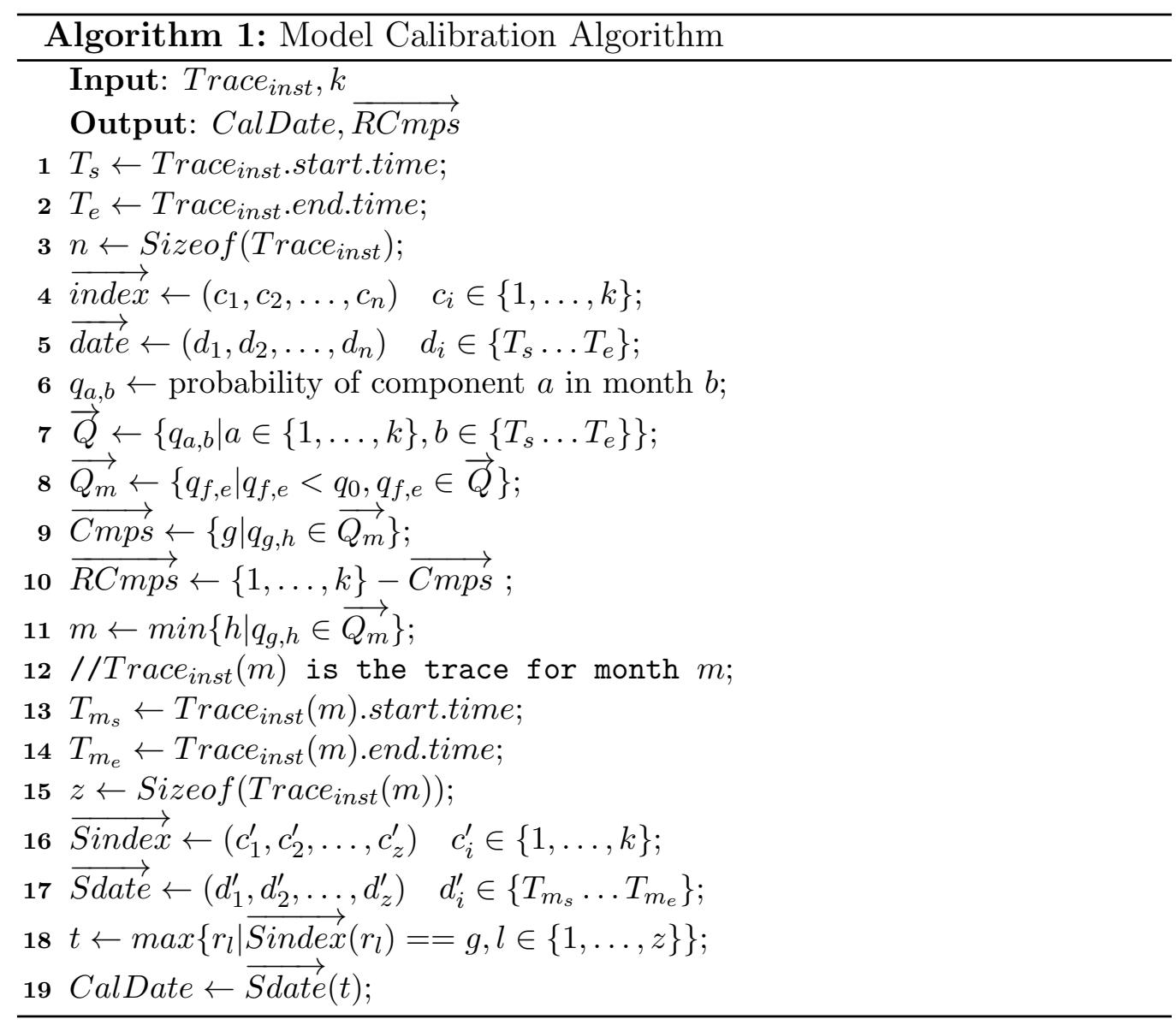


less than $q_{0}$ (line 4-8). $q_{0}$ is a threshold value and we define it as low as 0.01 (i.e. $q_{0}=0.01$ ). The components that are not in this list are remaining

components $(\overrightarrow{R C m p s}$ in line 10$)$. The first month in the list of $\overrightarrow{Q_{m}}$ is the calibration month, called $m$ (line 11). Finally, the last occurrence of the component(s) in month $m$ would be the calibration date (CalDate), which is obtained in line 13-19.

The results of applying this algorithm for all SIs in eu-west and us-east data centers are presented in Table 12 where all calibration dates are in July. The remaining components can be inspected in the fifth column ( $\vec{p}$ of the inter-price time model) of Table 10 and Table 11, where the component(s) with higher probability remain(s) beyond calibration date. For instance, the third component of the inter-price time model for m2.4xlarge in euwest with probability of 0.8 (1-0.063-0.137) remains after 15-July where the mean and variance are 1.279 and 0.022 hours, respectively. The graphical demonstration of Figure 8(b) can confirm the correctness of this algorithm, where component 3 implies a cluster around the mean value of 1.279 hours.

The last step of the model calibration is probability adjustment where the probability of remaining component(s) must be scaled up to one. This adjustment can be done by the following formula:

$$
p_{j}=\frac{p_{j}}{\sum_{\forall i} p_{i}} \quad i, j \in \overrightarrow{R C m p s}
$$

In other words, in the calibrated model for each SI, we just change the probability of remaining component(s) after the calibration date. In the following section, we investigate the accuracy of the calibrated model with respect to the real price history as well as the non-calibrated model.

\section{Model Validation}

In order to validate the proposed model, we implemented a discrete event simulator using CloudSim [28]. The simulator has a general architecture of IaaS Cloud with capability of provisioning of on-demand and Spot instances for input workload. The simulator uses the model or the price history traces to run the input workload. We consider the case where the user requests for one VM from one type of SI and runs whole jobs on that VM. The total monetary cost of running the workload on an SI is the parameter to be considered. In this section, we only present the results for eu-west the validation results are the same for us-east and other data centers. 
Table 12: The results of model calibration in eu-west and us-east $(k=3)$.

\begin{tabular}{|c|c|c|c|c|}
\hline \multirow[t]{2}{*}{ Instances } & \multicolumn{2}{|c|}{ Calibration Dates } & \multicolumn{2}{|c|}{ Remaining Components } \\
\hline & eu-west & us-east & eu-west & us-east \\
\hline m1.small & 24-July & 25-July & 3 & 1,3 \\
\hline c1.medium & 15-July & 25-July & 1 & 1 \\
\hline m1.large & 15-July & 26-July & 3 & 2,3 \\
\hline m2.xlarge & 13-July & 27-July & 1 & 2 \\
\hline m1.xlarge & 23-July & 24-July & 1 & 1 \\
\hline c1.xlarge & 23-July & 26-July & 1 & 1,3 \\
\hline $\mathrm{m} 2.2 \mathrm{xlarge}$ & 23-July & 26-July & 1,2 & 2 \\
\hline m2.4xlarge & 15-July & 26-July & 3 & 2,3 \\
\hline
\end{tabular}

\subsection{Simulation Setup}

The workload that we use in our experiments is the workload traces from LCG Grid which is taken from the Grid Workloads Archive [29]. We use the first 1000 jobs of this trace as the input workload for the experiments which is long enough to reflect the behavior of spot price for different SIs. We assume that one EC2 compute unit is equivalent of a CPU core with capacity of $1000 \mathrm{MIPS}^{4}$. We also assume that all jobs can be executed on a single VM, so we do not have any parallel jobs. As such, the selected workload needs about two weeks ( $\approx 400$ hours) to complete on a single m1.small instance. For other instance types we consider the linear speedup with the computing capacity in terms of EC2 compute unit which are listed in Table 1. For each experiment, the results are collected for 50 simulation rounds.

Moreover, we assume a very high user's bid for each simulation (for example on-demand price) where we do not have any out-of-bid event in the execution of the given workload. We use the model with three components $(k=3)$ for both spot price and the inter-price time to show the trade offbetween accuracy and complexity. In our experiments, the results of the simulations are accurate with a confidence level of $95 \%$.

\footnotetext{
${ }^{4}$ Amazon mentioned that one EC2 compute unit has equivalent CPU capacity of a 1.0-1.2 GHZ 2007 Opteron or 2007 Xeon processor [2].
} 


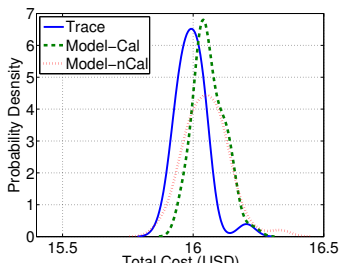

(a) m1.small

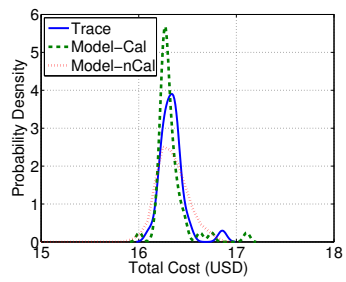

(e) m1.xlarge

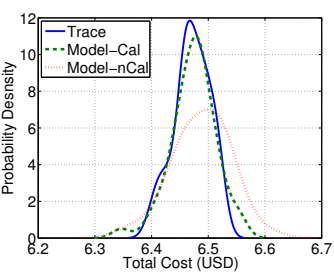

(b) c1.medium

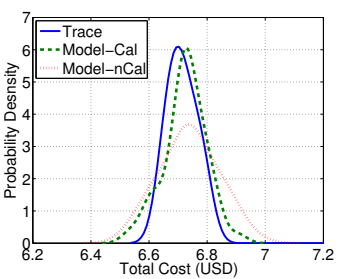

(f) c1.xlarge

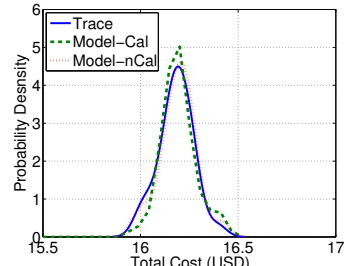

(c) m1.large

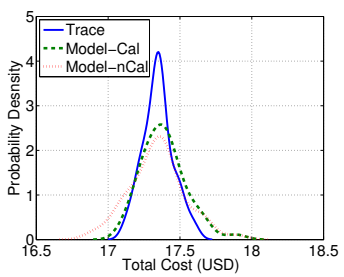

(g) m2.2xlarge

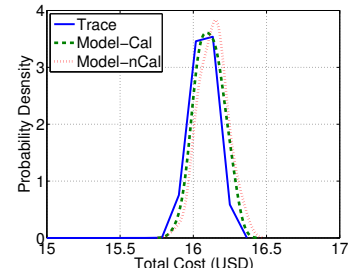

(d) m2.xlarge

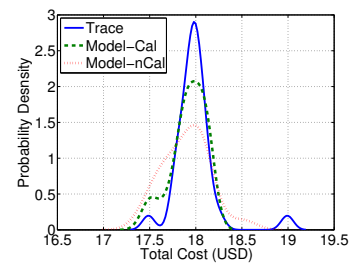

(h) m2.4xlarge

Figure 10: Model validation for all SIs in eu-west for the modeling traces (Feb-2010 to Nov-2010). 


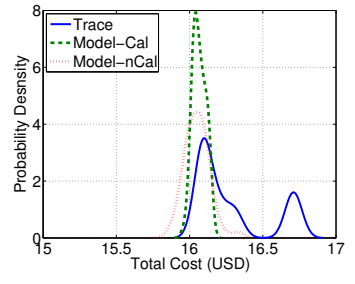

(a) m1.small

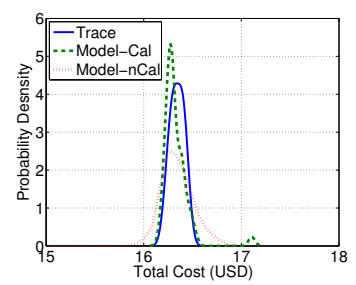

(e) m1.xlarge

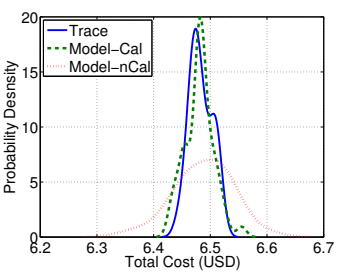

(b) c1.medium

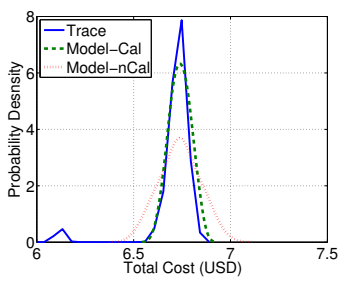

(f) c1.xlarge

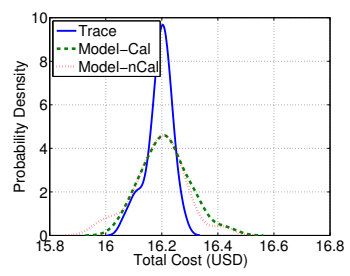

(c) m1.large

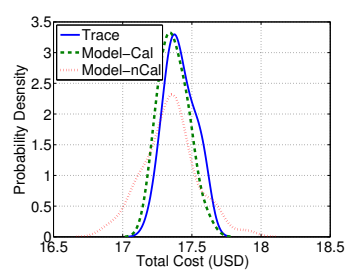

(g) m2.2xlarge

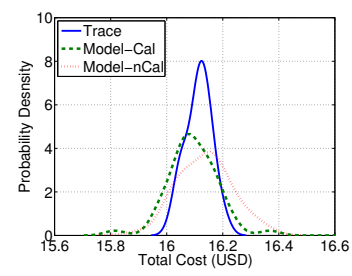

(d) m2.xlarge

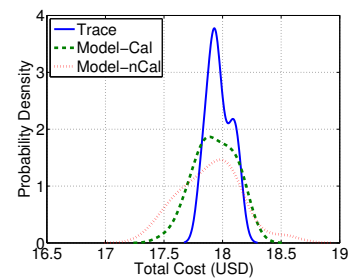

(h) m2.4xlarge

Figure 11: Model validation for all SIs in eu-west for the new traces (Dec-2010 to midFeb-2011). 


\subsection{Results and Discussions}

In the following, we present the results of two different set of experiments. First, we discuss the results of model validation where we have the price history that was included in the modeling process (i.e. Feb-2010 to Nov2010). Second, we report the results from model validation using a new price history which was not included in the modeling process. The new price history is from December 2010 till mid-February 2011.

Figure 10 shows the model validation results where the probability density functions of the total monetary cost to run the given workload have been plotted for all types of SIs. In each plot, Trace, Model-Cal, and Model-nCal refer to the result of using the real price history, the model after calibration and the model before calibration, respectively. Based on these figures, the proposed models match the real trace simulations with a high degree of accuracy, specially for the calibrated models. As we can see in these plots, in all cases the calibrated models are the better match with the trace simulations. As we expect, there are discrepancies in the model and trace simulation results for m1.small instance. However, the mean total cost for running the given workload for all SIs is very accurate where the maximum relative error is less than $3 \%$ for both calibrated and non-calibrated model, respectively.

Additionally, we report the model validation results where we use the new price history from December 2010 to mid-February 2011 to see the quality of the models for the future traces. The result of the simulations for the new price history are plotted in Figure 11. The results reveal that our models with three components still conform to the trace simulation results, except for m1.small instance. As mentioned earlier, spot price for m1.small instance is hard to fit and this is the reason of this inaccuracy. This means that for m1.small, we should use the model with more components (e.g. $k=4)$ to get the better accuracy. The calibrated models again match better with the trace simulations in comparison to the non-calibrated models for all SIs. Besides, the maximum relative error of the mean total cost for all SIs is less than $4 \%$ for both calibrated and non-calibrated model. Therefore, the proposed models are accurate enough for the new price history as well.

\section{Conclusions}

We considered the problem of discovering models for Spot Instances in Amazon's EC2 data centers for spot price and the inter-price time. The main motivation behind this is to explore characterization of SIs that is essential in 
the design of stochastic scheduling algorithms and fault tolerant mechanisms (e.g. checkpointing and replication algorithms) in Cloud environments for spot market. We studied the price patterns of the Amazon's data centers for a one year period and provided a global statistical analysis to get a better understanding of these patterns. Based on this understanding and observed bi-modality in probability densities, we proposed a model with mixture of

Gaussians distribution with 3 or 4 components for eight different types of SIs. The proposed model is validated through simulations, which reveals that our model predicts the total price of running jobs on spot instances with a good degree of accuracy. We believe that the proposed model are helpful for researchers and users of spot Instances in Amazon's EC2 data centers as well as other IaaS Cloud providers that look forward to offer such a service in the near future.

In future work, we intend to consider the user's bid as another parameter and investigate how it can affect the distribution of failures. Moreover, we would like to design a brokering solution to utilize different types of Cloud resources to optimize the monetary cost as well as job completion time. This can be easily realized by extending scheduling or resource provisioning components of cloud application platforms such as Aneka [30] and incorporating models and techniques proposed in this paper.

\section{Acknowledgments}

The authors would like to thank William Voorsluys for useful discussions. This work supported by a Discovery Project research grant form the Australian Research Council (ARC).

\section{References}

[1] J. Varia, Cloud Computing: Principles and Paradigms, Wiley Press, 2011, Ch. 18: Best Practices in Architecting Cloud Applications in the AWS Cloud, pp. 459-490.

[2] Amazon Inc., Amazon Elastic Compute Cloud (Amazon EC2), http: //aws.amazon.com/ec2.

[3] S. Yi, D. Kondo, A. Andrzejak, Reducing costs of spot instances via checkpointing in the amazon elastic compute cloud, in: 3rd IEEE International Conference on Cloud Computing, 2010, pp. 236 -243. 
[4] S. Yi, D. Kondo, A. Andrzejak, Monetary cost-aware checkpointing and migration on amazon cloud spot instances, IEEE Transactions on Services Computing (2011) 236-243.

[5] M. Mattess, C. Vecchiola, R. Buyya, Managing peak loads by leasing cloud infrastructure services from a spot market, in: 12th IEEE International Conference on High Performance Computing and Communications, 2010, pp. 180-188.

[6] F. M. Ortuno, U. Harder, A stochastic calculus model for the spot price of computing power, in: The UK Performance Engineering Workshop, UKPEW, 2010.

[7] K. Vanmechelen, W. Depoorter, J. Broeckhove, Combining futures and spot markets: A hybrid market approach to economic grid resource management, Journal of Grid Computing 9 (2011) 81-94.

[8] Cloud exchange website, http://cloudexchange.org/.

[9] Amazon Inc., Amazon Discussion Forums, https://forums.aws . amazon.com.

[10] B. Javadi, R. Buyya, Comprehensive statistical analysis and modeling of spot instances in public Cloud environments, Research Report CLOUDS-TR-2011-1, Cloud Computing and Distributed Systems Laboratory, The University of Melbourne (March 2011).

[11] S. Wee, Debunking real-time pricing in cloud computing, in: 11th IEEE/ACM International Symposium on Cluster, Cloud and Grid Computing (CCGrid), 2011, pp. $585-590$.

[12] O. A. Ben-Yehuda, M. Ben-Yehuda, A. Schuster, D. Tsafrir, Deconstructing Amazon EC2 spot instance pricing, in: 3rd IEEE International Conference on Cloud Computing Technology and Science (CloudCom), 2011, pp. 304-311.

[13] S. Chaisiri, R. Kaewpuang, B.-S. Lee, D. Niyato, Cost minimization for provisioning virtual servers in amazon elastic compute cloud, in: 19th IEEE International Symposium on Modeling, Analysis Simulation of Computer and Telecommunication Systems (MASCOTS), 2011, pp. 85 -95 . 
[14] W. Voorsluys, S. K. Garg, R. Buyya, Provisioning spot market cloud resources to create cost-effective virtual clusters, in: 11th International Conference Algorithms and Architectures for Parallel Processing (ICA3PP), 2011, pp. 395-408.

[15] A. Andrzejak, D. Kondo, S. Yi, Decision model for cloud computing under SLA constraints, in: 18th IEEE/ACM International Symposium on Modelling, Analysis and Simulation of Computer and Telecommunication Systems (MASCOTS), 2010, pp. 257-266.

[16] M. Mazzucco, M. Dumas, Achieving performance and availability guarantees with spot instances, in: 13th IEEE International Conference on High Performance Computing and Communications (HPCC), 2011, pp. $296-303$.

[17] N. Chohan, C. Castillo, M. Spreitzer, M. Steinder, A. Tantawi, C. Krintz, See spot run: using spot instances for MapReduce workflows, in: the 2nd USENIX conference on Hot topics in cloud computing, HotCloud'10, 2010, pp. 7-7.

[18] Q. Zhang, E. Gurses, R. Boutaba, J. Xiao, Dynamic resource allocation for spot markets in clouds, in: 11th USENIX conference on Hot topics in management of Internet, cloud, and enterprise networks and services, Hot-ICE'11, USENIX Association, Berkeley, CA, USA, 2011, pp. 1-1.

[19] Q. Zhang, Q. Zhu, R. Boutaba, Dynamic resource allocation for spot markets in cloud computing environments, in: 4th IEEE International Conference on Utility and Cloud Computing (UCC), 2011, pp. 178 -185.

[20] M. R. Rahman, Y. Lu, I. Gupta, Risk aware resource allocation for clouds, Technical Report 2011-07-11, University of Illinois at UrbanaChampaign (July 2011).

[21] D. G. Feitelson, Workload Modeling for Computer Systems Performance Evaluation, e-Book, http://www.cs.huji.ac.il/ feit/wlmod/, 2011.

[22] H. Li, Realistic workload modeling and its performance impacts in largescale escience grids, Parallel and Distributed Systems, IEEE Transactions on 21 (4) (2010) $480-493$. 
[23] D. Kondo, B. Javadi, A. Iosup, D. H. J. Epema, The Failure Trace Archive: Enabling comparative analysis of failures in diverse distributed systems, in: 10th IEEE/ACM International Conference on Cluster, Cloud and Grid Computing (CCGRID), 2010, pp. 398-407.

[24] D. Kondo, A. Andrzejak, D. P. Anderson, On correlated availability in internet distributed systems, in: 9th IEEE/ACM International Conference on Grid Computing (Grid 2008), 2008, pp. 276-283.

[25] C. Fraley, A. E. Raftery, Model-based clustering, discriminant analysis, and density estimation, Journal of the American Statistical Association 97 (458) (2002) 611-631.

[26] H. Li, M. Muskulus, L. Wolters, Modeling correlated workloads by combining model based clustering and a localized sampling algorithm, in: Proceedings of the 21st annual international conference on Supercomputing, ICS '07, ACM, New York, NY, USA, 2007, pp. 64-72.

[27] B. Javadi, D. Kondo, J.-M. Vincent, D. P. Anderson, Mining for statistical availability models in large-scale distributed systems: An empirical study of SETI@home, in: 17th IEEE/ACM International Symposium on Modelling, Analysis and Simulation of Computer and Telecommunication Systems (MASCOTS), 2009, pp. 1-10.

[28] R. N. Calheiros, R. Ranjan, A. Beloglazov, C. A. F. De Rose, R. Buyya, CloudSim: a toolkit for modeling and simulation of cloud computing environments and evaluation of resource provisioning algorithms, Software: Practice and Experience 41 (1) (2011) 23-50.

[29] A. Iosup, H. Li, M. Jan, S. Anoep, C. Dumitrescu, L. Wolters, D. H. J. Epema, The Grid Workloads Archive, Future Generation Computer Systems 24 (7) (2008) 672-686.

[30] C. Vecchiola, R. N. Calheiros, D. Karunamoorthy, R. Buyya, Deadlinedriven provisioning of resources for scientific applications in hybrid clouds with Aneka, Future Generation Computer Systems 28 (1) (2012) $58-65$. 